\title{
Bruceanol Agent
}

National Cancer Institute

\section{Source}

National Cancer Institute. BruceanolAgent. NCI Thesaurus. Code C1975.

A group of quassinoid phytochemicals extracted from species of the plant Brucea with potential antineoplastic activity. (NCI04) 